\section{COBIT, Herramienta de Control en la Gestión Empresarial}

\author{
COBIT, Control Tool in Business Management
}

\section{RESUMEN}

En concordancia con las nuevas necesidades y tendencias de los negocios como: "COSO (Committee of Sporisoring Organisation of the treadway Commission Internal Control-Integrated Framework, 1992 en los EUA, Cadhuay en el Reino Unido, CoCo en Canada y King en Sudáfrica, y la coexistencia de modelos de control en los niveles de tecnología de información como: Security Code of conduct del DTT( Departamento de Industria y Comercio , Reino Unido ) y el Security Handbook de Nist( Nacional Institute of Standards and technology, EUA)."

Ante la necesidad que los modelos mencionados no proporcionen un modelo de control completo y utilizable en a gestión empresarial, la ISACF (Information Systems Audit an Control foudation) y El ITGI (Governance Institute), desarrollan COBIT para cubrir el vacío pre-existente para logar los fines planeados en las organizaciones, con el desarrollo con el nuevo punto de vista que planetan las nuevas tecnologías de informacion. COBIT integra y consolida normas y reglamentos ya señaladas por las instituciones mencionadas.

Palabras claves: Herramientas; Control; Gestión; COBIT.

\begin{abstract}
In line with new business needs and trends such as: "COSO (Committee of Sporisoring Organization of the treadway Commission Internal Control-Integrated Framework, 1992 in the US, Cadhuay in the United Kingdom, CoCo in Canada and King in South Africa, and the coexistence of control models at the information technology levels such as: Security Code of conduct of the DTT (Department of Industry and Commerce, United Kingdom) and the Security Handbook of Nist (National Institute of Standards and technology, USA)."

Given the need that the aforementioned models do not provide a complete and usable control model in business management, the ISACF (Information Systems Audit an Control foudation) and ITGI (Governance Institute), develop COBIT to cover the pre-existing vacuum to achieve the aims planned in the organizations, with the development with the new point of view that the new information technologies plan. COBIT integrates and consolidates rules and regulations already indicated by the aforementioned institutions. Keywords: Tools; Control; Management; COBIT.
\end{abstract}

\section{Félix Armando Rivera León ${ }^{1}$}

Felix_arl@hotmail.com

\section{Félix A. Rivera Sandoval ${ }^{1}$}

riverasandovalfelix@gmail.com

${ }^{1}$ Universidad Nacional Mayor de San Marcos, Facultad de Ciencias Administrativas. Lima, Perú 


\section{INTRODUCCIÓN}

No todos los modelos de control general como: COSO; CoCo; King, y su coexistencia con los controles de tecnología de información como:"Security Code of conduct del DTT; Security Handbook de Nist", nos facilitan una guía completa sobre la Tecnología de Información (TI), influenciando en el contexto de los negocios. Para cubrir este vacío la información "Systems Audit and Control Foundation (ISACF)" y "Governance Institute (ITGI)" desarrollan el Control Objective for Information and related Technology (COBIT) (2006), "como una base que está ligada a los objetivos propios del negocio empresarial relacionándose estrechamente al enfoque tecnológico de información" (Pag.11).

A pesar de la realidad de la tecnología de acuerdo a casos puntuales, el COBIT establece con apoyo de las técnicas internacionales, es por ello que las mejores practica para la seguridad, calidad, eficiencia, donde la eficiencia técnica de información (TI), son indispensables. En ese entender COBIT (2006), expresa:

Para relacionar el TI con el negocio, requiere identificar riesgos, entregar valor al negocio, gestionar recursos y medir el desempeño, el cumplimiento de metas y el nivel de madurez de los procesos de la organización. La misión COBIT es para investigar, desarrollar, hacer público y promover un juego autoritario, actualizado e internacional de objetivos de control de TI generalmente aceptados para el empleo cotidiano por directores comerciales y auditores. Así mismo; el concepto fundamental del marco referencial COBIT se refiere a que el enfoque del control en TI se lleva a cabo visualizando la información necesaria para dar soporte a los procesos del negocio, considerando a la información como el resultado de la aplicación combinada de recursos relacionados que deben ser administrados. Para satisfacer los objetivos del negocio, la información necesita concordar con criterios a los que COBIT hace referencia como requerimientos de negocio para la información. Al establecer la lista de requerimientos, COBIT combina los principios contenidos en los modelos existentes y conocidos. Los criterios con los que se evaluará la información requerida son:
- "Requerimiento de Calidad: Calidad, Costo, Oportunidad (en tiempo indicado)".

- "Requerimientos Fiduciarios: Efectividad y Eficiencia operacional; confiabilidad de los reportes financieros; cumplimiento de la Leyes y Regulaciones".

- "Requerimientos de Seguridad: Confidencialidad, Integridad, Disponibilidad." (COBIT, 2006, p.11)

COBIT integra y consolida normas y reglamentos existentes (COBIT. 2006. pag. 11), como:

- Estándares Profesionales: "COSO (Committee of Sponsoring Organizations of the Treadway Commisssion Internal Control-Integrated Framework, 1992) en los EUA, Cadbury en el Reino Unido, CoCo en Canadá y King en Sudáfrica, GAO, IFAC, ISAC, Estándares CPA, etc".

- Estándares Técnicos: "ISO (9000-3) EDIFACT, etc".

- Códigos de Conducta: "OECD, ISACA, etc".

- Prácticas y requerimientos de la Industria.

COBIT como herramienta es autónoma de la plataforma técnica de TI, es por ello que la organización accede la aceptación de "KPIS" (key performance indicators) entre ellos (normas, reglas, etc) reconocida prioritariamente para futuros progresos que se ejecutan en la estructura (Netconsult.com)

Actualmente "COBIT" ha publicado cuatro ediciones:

- 1996, la primera, para aplicaciones de negocios publicado por ISACF.

- En el 1998, la segunda edición agrego "Lineamientos de gestión". Con un conjunto de herramientas de aplicación y alto nivel y control de objetivos detallados que se agrega.

- En el 2000, la tercera edición fue puesta en libertad: que incluye las directrices de gestión. "Instituto de Administración de las Tecnologías de la Información (ITGI)", en 1992 se convierte en el primer editorial de marco; en 2003, versión que estuvo disponible. 
- En 2005, la cuarta edición tuvo una publicación inicial; Luego mejorada significativamente en COBIT mediante la consolidación de la mayoría de los libros separados en un solo volumen para facilitar su uso.

En mayo de 2007. La revista actual 4.1 fue lanzado, ofreciendo una descripción simplificada de "Objetivos" y una cascada de procesos y (bidireccional) las relaciones entre los "Negocios", los "Objetivos de TI", y "Procesos de TI" COBIT y otras normas.

COBIT. (2006); está diseñado de la siguiente manera:

\section{Administración:}

"Para ayudarlos a lograr un balance entre los riesgos y las inversiones en control en un ambiente de tecnología de información frecuentemente impredecible".

\section{Usuario:}

"Para obtener una garantía en cuanto a la seguridad y controles de los servicios de tecnología de información proporcionados internamente o por terceras partes".

3. Auditores de sistemas de información: "Para dar soporte a las opiniones mostradas a la administración sobre los controles internos" (pag 7).

\section{OBJETIVOS}

El presente articulo tiene como objetivo coadyuvar a los profesionales en Tecnología de Información, como a los especialistas en Auditoria Contable / Administración acerca de las buenas prácticas en el control y la administración en TI, para evaluar las TI existentes como soporte a la administración en su conjunto. Razón por la cual COBIT es una herramienta que coadyuva a la dirección en las empresas por el logro de su gestión, bajo las exigencias de control, aspectos técnicos, así como los riesgos del negocio, permitiendo una habilitación de desarrollo con políticas claras y de buenas prácticas en las TI. El enfoque de COBIT, es proporcionar indicadores de control que permitan poseer referencias definidas, bajo la aceptación y liderazgo de las empresas comerciales, gubernamentales y profesionales.

Así mismo, "COBIT" estimula el empleo de políticas y buenas prácticas para la seguridad y control en TI.
Por lo tanto, COBIT, busca satisfacer las necesidades de la administración (Eficiencia y Eficacia). Regulación, Control, Seguridad y Estrategia.

\section{ARGUMENTACIÓN}

El éxito de las TI depende del grado de satisfacción que pueda lograr en los contextos de soporte a:

- "Los requerimientos del negocio".

- "Organización de las actividades de TI".

- "Los principales recursos de TI".

- "Los objetivos de control gerencia".

COBIT enfoca prioritariamente la orientación del negocio alineando metas del negocio con los objetivos de las TI, logrando indicadores y parámetros de madurez. E identificando responsabilidades asociadas de los usuarios con procesos del negocio y las TI.

Consideraciones necesarias que ayuden a tener un buen manejo de los recursos empresariales.

Esta dirigido en primera instancia a los directivos, a los auditores, a los responsables del TI y las Entidades Estadales del control.

- Directivos

- Coadyuvar en el equilibrio de los posibles riesgos existente en las inversiones en el control dentro de los parámetros demarcados por TI

- En la toma de decisiones eficaces, ayudando en las definiciones de planes estratégicos a nivel de TI

- En la evaluación del entonces presentes y futuros

- Usuarios

- Garantizar seguridad y controles en los servicios de TI proporcionados.

- Los auditores

- El logro de igualar temas sobre el control de TI en cuanto a la infraestructura de la empresa

- Como soportes de opinión elevadas a la administración sobre los controles internos 
- Los responsables del TI

- En el logro de identificación de controles que se requieren en sus áreas

\section{- Entidades Estatales de control}

- Coadyuvar en las exigencias mínimas de desarrollo y control

En las organizaciones, la información y las TI que soportan, expresan activos más importantes. Se amalgaman profundamente, la plataforma del usuario con redes locales o amplias, cliente, servidor y equipos Mainframe, se entienden las claras ventajas que esta tecnología brinda. Las organizaciones exitosas administran los riesgos vinculados a la implementación de nuevas tecnologías, por ello la administración debe conocer de manera precisa las oportunidades y limitaciones de la implementación de TI.

Al tomar decisiones sobre una inversión de para TI y lograr un equilibrio entre riesgos e inversión de control, la administración requiere de un marco referencial de evaluación en seguridad y control de TI. Consideraciones temáticas que se han tomado procedentes de: ITSEC, TSEC, ISO 9000 entre otras, frecuentemente se requiere de una base general en la que se establezcan prácticas. Los auditores son los principales responsables en la estandarización de procesos, producto de su experiencia. Frente a la necesidad de informar a la alta dirección en las empresas, su punto de vista para los controles internos pre-existentes. Al no contar con un marco referencial programático en sus labores, se convierten en tareas demasiado complicadas. A dado lugar a que la Comisiones nombradas emitan COBIT como una herramienta básica de avaluación en el contexto administrativo y los soportes de información (TI).

Como premisa, COBIT, tiene en cuenta que el principal y fundamental logro es satisfacer los objetivos de la organización de la empresa, busca combinar principios contenidos en los modelos referenciales existentes, ya mencionados.

Los recursos TI identificados para las necesidades COBIT pueden identificarse y definirse a continuación:

- "Datos. - Los elementos de datos en su mas amplio sentido, (por ejemplo, exter- nos e internos), estructurados o no estructurados; gráficos; sonidos".

- "Aplicaciones. - Suma de procedimientos programados, como: manuales".

- "Tecnología. - Hardware; software; sistemas operativos; sistemas de administración en la base de datos; redes; multimedia".

- "Instalaciones. - Proporcionan soporte a los sistemas de información".

- $\quad$ "Personal. - Habilidades; conocimiento; conciencia y productividad para planear; organizar; adquirir; entregar; soportar; monitorear servicios y sistemas de información".

Los procesos del negocio requieren información, la que es proporcionada por el TI. Para ser satisfactorios y razonables deben ser definidos, implementados y monitoreados bajo indicadores de control adecuados.

Por lo tanto, debe existir un marco referencial que les proporcione soporte a todos los procesos del negocio que satisfaga los requerimientos e impacte en los recursos de TI (Ver Figura 1).

En el cuadro se puede observar la manera que el COBIT, se interrelación con los procesos del TI, principalmente se focalizado en el Control, medidas de control evaluados por las metas y actividades, directrices y practicas de control, todo esto tiene fines específicos, los cuales son: mejorar los procesos, la eficiencia de los recursos, la adaptabilidad al cambio y asumir riesgos.

El estudio que se realice estará enfocado principalmente hacia los procesos que tiene el COBIT, además de su investigación y desarrollo que son aplicadas en las empresas.

COBIT permite salvar brechas entre los riesgos empresariales, necesidades de seguimiento y control, así como aspectos técnicos. Proporciona buenas prácticas en el marco referencial de dominios y procesos, presentando una estructura lógica.

COBIT es una herramienta informática que se utiliza para controlar de manera eficiente todos los recursos, y en donde se analiza procesos que lo involucran, siendo estos, idealizados por ISACF y ITGI: 


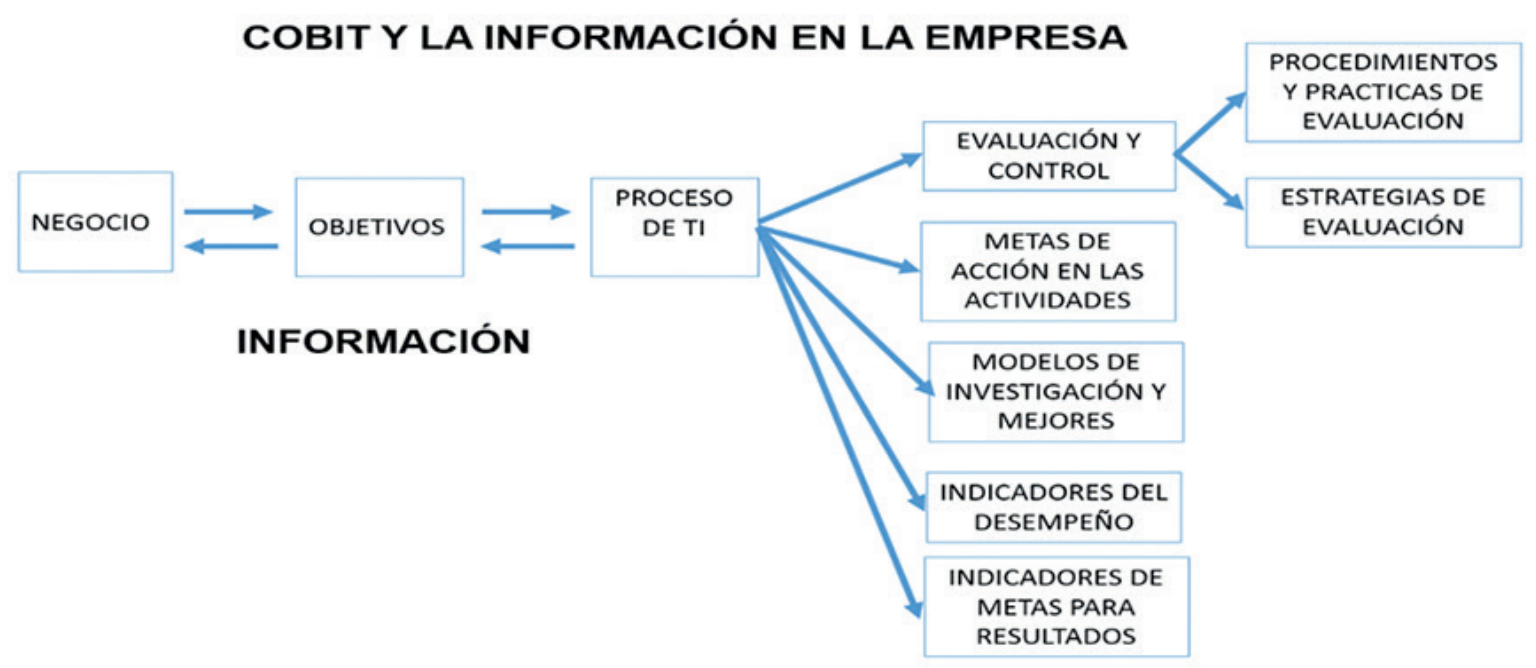

Figura 1. Cobity la Información en la empresa. Fuente: Elaboración propia.

- "Planear y Organizar"

- "Adquirir e Implementar"

- "Entregar y Dar Soporte"

- "Monitorear y Evaluar"

Dominios que, en base a la información, busca en sus contenidos: efectividad, eficiencia, confidencialidad, integridad, disponibilidad, cumplimiento, confiabilidad; procesados a través del TI de la empresa mediante sus recursos, datos, aplicaciones, tecnología, instalaciones, recursos humanos.

COBIT plantea los cuatro dominios señalados, proponiendo análisis y evaluación en forma continua y permanente sobre estos, visualizando en cada uno de ellos lo siguiente:

\section{Planeación y Organización}

P01 Definir un Plan Estratégico de TI

P02 Definir la Arquitectura de Información

P03 Determinar la dirección tecnológica

P04 Definir la Organización y Relaciones de TI

P05 Manejar la Inversión en TI

P06 Comunicar las directrices gerenciales

P07 Administrar Recursos Humanos

P08 Asegurar el cumplir Requerimientos Externos

P09 Evaluar Riesgos
P010 Administrar proyectos

P011 Administrar Calidad

\section{Adquisición e implementación}

A11 Identificar Soluciones

A12 Adquisición y Mantener Software de Aplicación

A13 Adquirir y Mantener Arquitectura de TI

A14 Desarrollar y Mantener Procedimientos relacionados con TI

A15 Instalar y Acreditar Sistemas

A16 Administrar Cambios

\section{Servicio y Soporte}

DS1 Definir niveles de servicio

DS2 Administra Servicios de Terceros

DS3 Administrar Desempeño y Calidad

DS4 Asegurar Servicio Continuo

DS5 Garantizar la Seguridad de Sistemas

DS6 Identificar y Asignar Costos

DS7 Capacitar Usuarios

DS8 Asistir a los Clientes de TI

DS9 Administrar la Configuración

DS10 Administrar Problemas e Incidentes

DS11 Administrar Datos

DS12 Administrar Instalaciones 
DS13 Administrar Operaciones

\section{Monitoreo}

M1 Monitorear los procesos

M2 Evaluar lo adecuado del control interno

M3 Obtener aseguramiento independiente

M4 Proveer auditoría independiente

Cada dominio del negocio posee un conjunto de procesos e indicadores de gestión y desarrollo, permite evaluar el grado de satisfacción sobre las necesidades del negocio, correlacionados con la TI. Desarrollando un servicio de soporte, coadyuvado con:

- La intervención de gerencias del negocio alineando la planeación estratégica del TI con las necesidades en las organizaciones actuales, como futuras.

- La comprensión de las capacidades actuales del TI.

- Estandarización de prioridades para los objetivos del negocio que cuantifique los requerimientos empresariales.

- Los porcentajes de objetivos en las TI y sus planes acción dan soporte al correspondiente plan estratégico del negocio.

- Los porcentajes de proyectos TI en los portafolios, se pueden rastrear hacia las acciones tácticas en la gestión empresarial.

- La elasticidad del tiempo entre las actualizaciones.

Consideraciones que COBIT pone en práctica lineamientos como los señalados, con sus objetivos de aplicación pertinentes.

\section{CONCLUSIÓN}

COBIT:

1. Realiza y se fundamenta mediante su orientación hacia el negocio, correlacionan las metas de la TI con lo que se quiere alcanzar en el negocio, identificando responsabilidades de los usuarios con procesos de negocio y del TI. Se basa en modelos de actividades por procesos, subdividiéndose en 34 actividades de acuerdo a las responsabilidades de planear y organi- zar; adquirir e implementar; servicio y soporte; monitorear, logrando una visión integral de la TI.

2. Uno de los roles de importancia en las empresas es: planear la información y la tecnología, por lo que las empresas establecen sus objetivos en el corto, mediano y largo plazo, considerando que una buena planificación y organización con la información y la tecnología son de gran utilidad para los resultados eficientes preestablecidos.

3. Contando con un buen plan estratégico logrado en el desarrollo de la organización es posible obtener objetivos preestablecidos, los que deberán tener un soporte de información necesaria permanentemente actualizada, poseer transparencia y control los que deben ser incorporados al plan estratégico durante la labor realizada, logrando un subsistema de información interna, así mismo, ya que todos los trabajadores al intervenir deberían tener conocimientos claros sobre el plan, los objetivos, la misión, visión, políticas etc.

4. Cabe señalar que las tecnologías de información TI, se encuentra dentro de todos los criterios mencionados como parte del desarrollo y eficiencia de las actividades a través de la fuerza laborar en la organización.

5. Los requerimientos y/o servicios de los usuarios y su disponibilidad, va a depender del dominio de entrega y dar soporte, dado que, es netamente operativo, lo que es de gran importancia al efectivo cumplimiento de las tareas y responsabilidades asignadas mediante el aseguramiento de las condiciones necesarias para que dichas tareas puedan ser llevadas a cabo de la mejor manera posible.

6. Es un marco de referencia, una herramienta, permite a la gerencia lograr técnicas y evaluar riesgos del negocio, y comunicar niveles de control a los usuarios.

7. La arquitectura de procesos y su enfoque orientado al ámbito empresarial , permite lograr una visión esférica de los conceptos de TI y de las decisiones a tomar. 


\section{Aplicación}

- López, C. (2015). "Sistema de Control basado en COBIT para la certificación SOX. Caso: AFP en Perú (Tesis de Máster en Dirección Estratégica de Tecnologías de la Información). Universidad de Piura. Facultad de Ingeniería. Piura, Perú", nos menciona que: "La tesis tiene como objetivo establecer un marco de trabajo estándar y homologado en la organización para la gestión de tecnología, así como también de la gestión de riesgos tecnológicos, con la finalidad de implementar controles que permita la certificación Ley Sarbanes-Oxley (SOX). Para ello, el estudio se aplica a una Empresa Administradora de Pensiones (AFP) y a través del método del test al área de tecnología se busca detectar la situación actual de gobierno TI. La investigación considera tres pilares fundamentales para la implementación de SOX: Cobit (Guía a seguir sobre trabajo de dominios y procesos), ISO 27002 (buenas prácticas a seguir sobre seguridad de la Información) e Itil (Informatión Technology Infraestructure Library proporciona un planteamiento sistemático para la provisión de servicios de TI con calidad). Finalmente, se realiza una autoevaluación para poder mantener los controles en el tiempo."

- RECSA (2018),... implementa el COBIT en Argentina en lo que se denomina: "PROCESOS DE INSTALACION DE COBIT, constituye la redefinición de los procesos internos de TI (técnicas de información), basados en los dominios de COBIT. En la base a la agrupación lógica de los procesos que intervienen en el ciclo de vida de los procesos de TI de RECSA Argentina se redefinieron los mismos en base a los expuestos por $\mathrm{CO}$ BIT agrupados en los diferentes dominios del marco". Es parte del plan de mejoramiento integral el servicio al cliente, con el objetivo de optimizar la Tecnología Informática, negocio, su entrega de valor y a la vez administrar riesgos y los recursos de manera eficaz y eficiente. RECSA implementó el COBIT en Argentina.

- En el ámbito de la docencia: el Colegio de Contadores de Lima (2000), implementó cursos de especialización con respecto al COBIT.

- Así mismo la "ESCUEla NACIONAL DE CONTROL de la CONTRALORIA GENERAL DE LA REPUBLICA" realizó Cursos: "COBIT, Implementación de Control Interno para Tecnologías de Información".

\section{REFERENCIAS BIBLIOGRÁFICAS}

Benal R. y Coltell O. (1996). Auditoria de los Sistemas de Informacion. Universidad Politécnica de Valencia.

COBIT, Competitividad en TI para los negocios: Emitido por ASSENTI Tendencias 2006.

COBIT, Directrices de Auditoria, (1998). 2da. Edición. Emitido por el Comité Directivo de COBIT y la Information Systems Audit and Control Foundation. (ITACA).

COBIT, Marco Referencial. 3a Edición. Emitido por el Comité Directivo de COBIT y EI Governance Institute.

Fernando Ferrer Olivares (2012). COBIT; Herramienta de IT Govemance. CISA - presidente de ISACA - Colombia

Fernando Izquierdo Duarte (2011). Administración de riesgos de TI. CISA - Ing. De Sistemas.

ISACAF (2000). COBIT. Audit Guidelines. $3^{\text {rd }}$ ed. ISACA, Rolling Meadows, IL (USA)

Koung Javier (2000). Seguridad, Control y Auditoria de las Tecnologías de Información. Editorial: Management Advisory Publications.

Peña, Eloy (2002). "Evolución de los sistemas de información y su auditoria”. Editorial: Auditores Eloy Peña

Peña, Eloy (2002). Programa de Auditoria Informática. Editorial: Auditores Eloy Peña. 